\title{
Classifying links under fused isotopy
}

\author{
ANDREW FISH and EBRU KEYMAN \\ School of Computing, Mathematical and Information Sciences, University of Brighton, UK \\ Andrew.Fish@brighton.ac.uk \\ Department of Mathematics, Middle East Technical University, Ankara, Turkey \\ ekeyman@metu.edu.tr
}

\begin{abstract}
All knots are fused isotopic to the unknot using a process known as virtualization. We extend and adapt this process to show that, up to fused isotopy, classical links are classified by their linking numbers.
\end{abstract}

Keywords: Fused isotopy, linking numbers

\section{Introduction}

Classical braids and links have been generalized to the virtual category [8] - adding virtual crossings and extending isotopy to allow the virtual analogues of the classical Reidemeister moves. The forbidden moves $F_{o}$ and $F_{u}$, shown in Figure 1 are not allowable under virtual isotopy. Extending virtual isotopy in the virtual braid group $V B_{n}$ to allow the $F_{o}$ move gives rise to the welded braid group $W B_{n}$, which has been shown to be isomorphic to $P C_{n}$, the group of automorphisms of the free group on $n$ elements of permutation-conjugacy type [3]. Allowing both of the forbidden moves $F_{o}$ and $F_{u}$ gives rise to fused isotopy [8]. That is, two virtual links $L_{1}$ and $L_{2}$ are called fused isotopic if $L_{2}$ can be obtained from $L_{1}$ by a finite sequence of Reidemeister moves, virtual moves and $F_{o}, F_{u}$ moves.
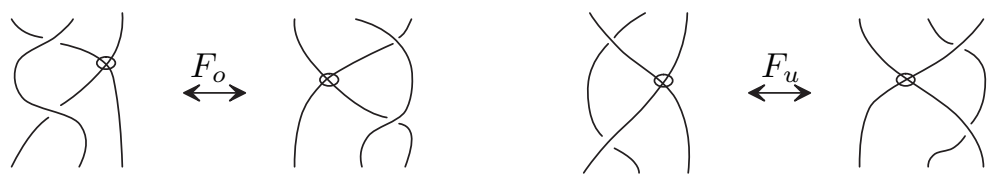

Figure 1: The forbidden moves

Let $\mathcal{K}$ be the space of classical links embedded in $S^{3}$ and let $\mathcal{V K}$ be the space of virtual links. Kauffman [8], and independently Goussarov-Polyak-Viro [5], have shown that $\mathcal{K}$ embeds into $\mathcal{V K}$. Let $f$ denote the natural inclusion of $\mathcal{V K}$ into the space of fused links $\mathcal{F} \mathcal{K}$. Then we have $\mathcal{K} \stackrel{i}{\hookrightarrow} \mathcal{V K} \stackrel{f}{\rightarrow} \mathcal{F} \mathcal{K}$, and when we refer to a classical link (under fused isotopy) we mean $f \circ i(L)$, the image of a link $L \in \mathcal{K}$ in the space $\mathcal{F} \mathcal{K}$.

In [7], Kanenobu showed that all knots are fused isotopic to the unknot. He showed that all of the classical crossings of a virtual knot can be virtualized; that is every classical crossing can be changed into a virtual crossing by applying a sequence of fused isotopy moves. However, crossings between different components of a link cannot be virtualized using the same methods. The following theorem from [7] provides us with allowable moves under fused isotopy which were used in the virtualization procedure.

Theorem 1. The moves $M_{1}, M_{2}$ and $M_{3}$, shown in Figure 2 can be realised by fused isotopy. 


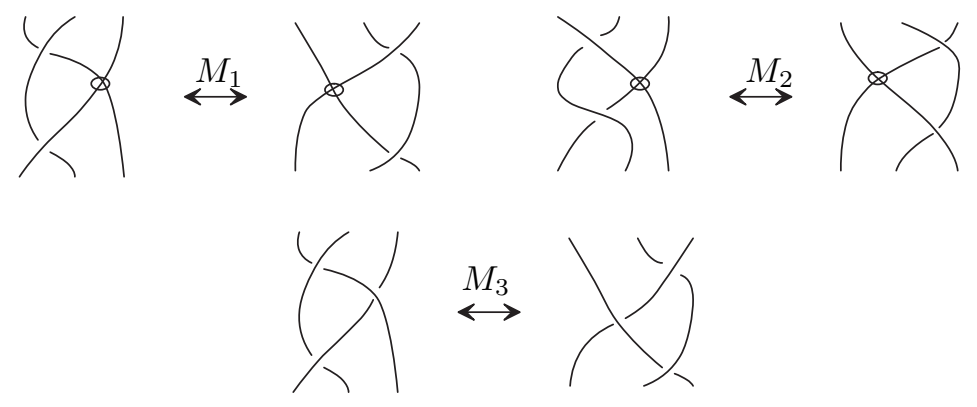

Figure 2: Allowable moves in fused isotopy

In [4] the authors showed that the Jones polynomial for welded and fused links is welldefined in a quotient of $\mathbb{Z}\left[A, A^{-1}\right]$ and observed that this polynomial depends only upon the linking number for links with two components. Inspired by this, we show that classical links, under fused isotopy, can be determined by the linking number of their components. Theorem 2. A classical link $L$ with $n$-components is completely determined by the linking numbers of each pair of components under fused isotopy.

The strategy that we use to prove Theorem 2 is to write $L$ as the closure of a braid $\alpha$ on $m$ strands (where $m \geq n$ ) and then to transform $\alpha$ into a pure braid $\beta$ on $n$ strands whose closure is also $L$. We show that $\beta$ depends only on the linking numbers of the components of $L$. This means that any classical link with the same linking numbers as $L$ can be obtained as the closure of $\beta$. We need some preliminaries before we proceed with the proof.

\section{Preliminaries}

Recall that an element of the pure braid group $P_{n}$ is an $n$-strand braid where the permutation induced by the strings is the identity. $P_{n}$ has a presentation with generators $A_{i, j}$ with $1 \leq i<j \leq n$ where

$$
A_{i, j}=\sigma_{j-1} \sigma_{j-2} \ldots \sigma_{i}^{2} \ldots \sigma_{j-2}^{-1} \sigma_{j-1}^{-1}=\sigma_{i}^{-1} \sigma_{i+1}^{-1} \ldots \sigma_{j-1}^{2} \ldots \sigma_{i+1} \sigma_{i} .
$$

Let $U_{k}$ be the subgroup of $P_{n}$ generated by $\left\{A_{i, k}: 1 \leq i<k\right\}$. Then every element of $P_{n}$ can be written in the unique normal form $x_{2} x_{3} \ldots x_{n}$, where $x_{k} \in U_{k}$ (see [1] for details). Define $B_{i, j}:=\sigma_{j-1} \ldots \sigma_{i+1} \sigma_{i}$ for $i<j$ and $B_{i, i}:=1$. Then by definition $A_{i, j+1}=B_{i, j}^{-1} A_{j, j+1} B_{i, j}$, and we can see from Figure 3 that for $k<i<j, B_{i, j}$ commutes with $A_{k, j+1}$ in $B_{n}$.

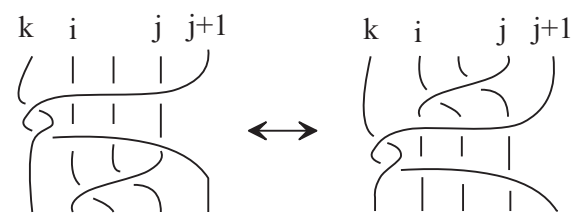

Figure 3: $A_{k, j+1} B_{i, j}=B_{i, j} A_{k, j+1}$

The virtual braid group on $n$-strands, $V B_{n}$, can be defined by adding extra generators $\tau_{i}$, for $1 \leq i \leq n$, corresponding to the virtual crossings, and relations corresponding to the virtual isotopy moves [6]. By adding the relations $\sigma_{i}^{-1} \tau_{j} \sigma_{i}=\sigma_{j} \tau_{i} \sigma_{j}^{-1}$ with $|i-j|=1$, 
to the virtual braid group, we obtain the fused braid group, $F B_{n}$. If $j=i+1$ the relation corresponds to the $F_{u}$ move, and if $i=j+1$ it corresponds to the $F_{o}$ move. The explicit realization of the moves $M_{1}, M_{2}$ and $M_{3}$ using $F_{o}$ and $F_{u}$ moves is shown in [7], and this gives rise to the following consequences in $F B_{n}$ :

$$
\begin{aligned}
M_{1}: & \sigma_{i} \tau_{j} \sigma_{i} & =\sigma_{j} \tau_{i} \sigma_{j} \\
M_{2}: & \sigma_{i}^{-1} \tau_{j} \sigma_{i}^{-1} & =\sigma_{j}^{-1} \tau_{i} \sigma_{j}^{-1} \\
M_{3}: & \sigma_{i} \sigma_{j}^{-1} \sigma_{i} & =\sigma_{j} \sigma_{i}^{-1} \sigma_{j},
\end{aligned}
$$

where $|i-j|=1$.

The following lemmas are used in the proof of Theorem 2 and the indices have been chosen to match the usage in the proof. Let $\sim$ denote the equivalence class generated by fused isotopy and let $\widetilde{U}_{k}=U_{k} / \sim$.

Lemma 1. In $F P_{n}$ we have:

$$
A_{j, j+1} A_{i, j+1} A_{j, j+1}^{-1}=A_{i, j+1} \text { where } 1 \leq i<j+1 \leq n .
$$

In other words, $A_{j, j+1}=\sigma_{j}^{2}$ is in the centre of $\widetilde{U}_{j+1}$.

Proof. Using the relations $\sigma_{j} \sigma_{j-1}^{-1} \sigma_{j}=\sigma_{j-1} \sigma_{j}^{-1} \sigma_{j-1}$ corresponding to an $M_{3}$ move, and $\sigma_{j} \sigma_{j-1} \sigma_{j}^{-1}=\sigma_{j-1}^{-1} \sigma_{j} \sigma_{j-1}$ corresponding to an $R_{3}$ move, we obtain

$$
\begin{aligned}
& \sigma_{j}^{2} \sigma_{j-1}^{-1} \sigma_{j}^{2} \sigma_{j-1} \sigma_{j}^{-2}=\sigma_{j}\left(\sigma_{j} \sigma_{j-1}^{-1} \sigma_{j}\right)\left(\sigma_{j} \sigma_{j-1} \sigma_{j}^{-1}\right) \sigma_{j}^{-1} \\
= & \sigma_{j}\left(\sigma_{j-1} \sigma_{j}^{-1} \sigma_{j-1}\right)\left(\sigma_{j-1}^{-1} \sigma_{j} \sigma_{j-1}\right) \sigma_{j}^{-1}=\sigma_{j} \sigma_{j-1}^{2} \sigma_{j}^{-1}
\end{aligned}
$$

Using Equation (2.3), we can compute

$$
\begin{array}{rlrl} 
& A_{j, j+1} A_{i, j+1} A_{j, j+1}^{-1} & \\
= & \sigma_{j}^{2} B_{i, j}^{-1} A_{j, j+1} B_{i, j} \sigma_{j}^{-2} & \\
= & B_{i, j-1}^{-1} \sigma_{j}^{2} \sigma_{j-1}^{-1} \sigma_{j}^{2} \sigma_{j-1} \sigma_{j}^{-2} B_{i, j-1} & & \text { by commutation in } B_{n} \\
= & B_{i, j-1}^{-1} \sigma_{j} \sigma_{j-1}^{2} \sigma_{j}^{-1} B_{i, j-1} & & \text { by Equation (2.3) } \\
= & \sigma_{j} B_{i, j-1}^{-1} \sigma_{j-1}^{2} B_{i, j-1} \sigma_{j}^{-1} & & \text { by commutation in } B_{n} \\
= & \sigma_{j} A_{i, j} \sigma_{j}^{-1} & \\
= & A_{i, j+1} . &
\end{array}
$$

Lemma 2. For every $1 \leq j+1<n$, the subgroup $\widetilde{U}_{j+1}$ of $F P_{n}$ is commutative.

Proof. Assume without loss of generality that $k<i$. Then

$$
\begin{array}{rlr}
A_{k, j+1} A_{i, j+1} & =A_{k, j+1} B_{i, j}^{-1} A_{j, j+1} B_{i, j} & \\
& =B_{i, j}^{-1} A_{k, j+1} A_{j, j+1} B_{i, j} \quad \text { by commutation in } B_{n} \\
& =B_{i, j}^{-1} A_{j, j+1} A_{k, j+1} B_{i, j} \quad \text { by Lemma! } \\
& =B_{i, j}^{-1} A_{j, j+1} B_{i, j} A_{k, j+1} & \text { by commutation in } B_{n} \\
& =A_{i, j+1} A_{k, j+1} . & \square
\end{array}
$$

Lemma 3. In $F B_{n}$ we have:

$$
A_{i, j+1} \tau_{j}=\tau_{j} A_{i, j} \quad \text { for } 1 \leq i \leq j-1 .
$$


Proof. Using the relations $\sigma_{j} \sigma_{j-1} \tau_{j}=\tau_{j-1} \sigma_{j} \sigma_{j-1}$ corresponding to an $F_{0}$ move, and $\sigma_{j-1}^{-1} \sigma_{j} \tau_{j-1}=\tau_{j} \sigma_{j-1} \sigma_{j}^{-1}$ corresponding to an $M_{1}$ move, we obtain

$$
\begin{array}{rlrl}
A_{i, j+1} \tau_{j} & =B_{i, j}^{-1} \sigma_{j}^{2} B_{i, j} \tau_{j} & \\
& =B_{i, j-1}^{-1} \sigma_{j-1}^{-1} \sigma_{j} \sigma_{j} \sigma_{j-1} B_{i, j-1} \tau_{j} & \\
& =B_{i, j-1}^{-1} \sigma_{j-1}^{-1} \sigma_{j} \sigma_{j} \sigma_{j-1} \tau_{j} B_{i, j-1} & & \text { by commutation in } V B_{n} \\
& =B_{i, j-1}^{-1} \sigma_{j-1}^{-1} \sigma_{j} \tau_{j-1} \sigma_{j} \sigma_{j-1} B_{i, j-1} & & \text { by an } F_{o} \text { move } \\
& =B_{i, j-1}^{-1-1} \tau_{j} \sigma_{j-1} \sigma_{j}^{-1} \sigma_{j} \sigma_{j-1} B_{i, j-1} & & \text { by an } M_{1} \text { move } \\
& =\tau_{j} B_{i, j-1}^{-1} \sigma_{j-1}^{2} B_{i, j-1} & & \text { by commutation in } V B_{n} \\
& =\tau_{j} A_{i, j} . &
\end{array}
$$

\section{Proof of Theorem 2}

Let $L$ be a classical link with $n$-components. By Alexander's Theorem, there exists $\alpha \in B_{m}$ with $m \geq n$, such that the closure of $\alpha$ is $L$. Chow [2] (or see page 22 of [1]) shows that every $\alpha$ can be written in the form $\alpha=x_{2} B_{k_{2}, 2} \ldots x_{m} B_{k_{m}, m}$ where $x_{i} \in U_{i} \leq P_{m}$ and $1 \leq k_{i} \leq i$. Let $\hat{\alpha}$ denote the closure of $\alpha$. If $m>n$ then we will construct $\beta \in B_{n}$ such that $\hat{\beta}$ is fused isotopic to $\hat{\alpha}$.

If $B_{k_{i}, i}=1$ for all $i=2, \ldots, m$ then $\alpha$ is a pure braid, which means that $m$ must be equal to $n$. So let us assume that $B_{k_{s}, s} \neq 1$, for some $s$, and that if $i>s$ then $B_{k_{i}, i}=1$. This means that the permutation induced by $\alpha$ is the identity on the strands $s+1, \ldots, m$. Therefore, each of these strands forms a separate component of the link $\hat{\alpha}$. Now, conjugating $\alpha$ with $B_{1, m}$ gives $B_{1, m}^{-1} \alpha B_{1, m}$, and as shown in Figure 4 the $(m-1)-$ strand of the original braid $\alpha$ becomes the $m$-strand of the new braid.

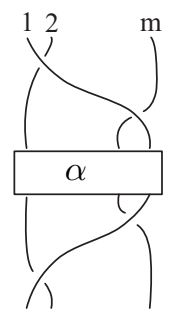

Figure 4: $B_{1, m}^{-1} \alpha B_{1, m}$

Thus if we conjugate $\alpha$ with $B_{1, m}(m-s)$ times, we get $\alpha^{\prime}=B_{1, m}^{s-m} \alpha B_{1, m}^{m-s}$ and the $s$-strand of $\alpha$ becomes the $m$-strand of $\alpha^{\prime}$. Since $\alpha^{\prime}$ is just a conjugate of $\alpha$ their closures are isotopic. Now write $\alpha^{\prime}=y_{2} B_{t_{2}, 2} \ldots y_{m} B_{t_{m}, m}$ with $y_{i} \in \widetilde{U}_{i}$. Then $B_{t_{m}, m} \neq 1$ and so by definition, $B_{t_{m}, m}=\sigma_{m-1} B_{t_{m}, m-1}$. A picture of $\alpha^{\prime}$ is shown in Figure 5 where $W=y_{2} B_{t_{2}, 2} \ldots y_{m-1} B_{t_{m-1}, m-1}$.

Since $\widetilde{U}_{m}$ is commutative (by Lemma 2), we can write

$$
y_{m}=\underbrace{A_{1, m}^{r_{1}} \ldots A_{m-2, m}^{r_{m-2}}}_{v_{m}} \underbrace{A_{m-1}}_{\begin{array}{c}
\sigma_{m-1}^{2 r_{m-1}} \\
A_{m-1, m}^{r_{m-1}}
\end{array}}
$$

for some $r_{1}, \ldots, r_{m-1}$. By definition, $A_{m-1, m}^{r_{m-1}}=\sigma_{m-1}^{2 r_{m-1}}$, and since $B_{t_{m}, m}=\sigma_{m-1} B_{t_{m}, m-1}$, we obtain $y_{m} B_{t_{m}, m}=v_{m} \sigma_{m-1}^{2 r_{m-1}+1} B_{t_{m}, m-1}$, where $v_{m}=A_{1, m}^{r_{1}} \ldots A_{m-2, m}^{r_{m-2}}$. 


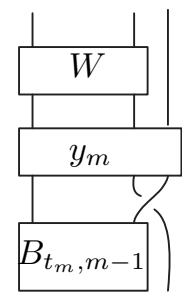

Figure 5: The braid $\alpha^{\prime}$

Since $W$ does not involve the $m$-strand and $y_{m}$ is a pure braid, Figure 5 shows that the $m$-strand and the other strand that is involved in the last occurrence (and hence in all of the previous occurrences) of $\sigma_{m-1}$ in $\alpha^{\prime}$ belong to the same component of $L=\hat{\alpha}^{\prime}$. Therefore, following the strategy in [7], we can virtualize all of the $2 r_{m-1}+1$ crossings in $\hat{\alpha}^{\prime}$ which correspond to $\sigma_{m-1}^{2 r_{m-1}+1}$ in $\alpha^{\prime}$. In doing so we have not changed the fused isotopy class of $L$ but we have obtained $L$ as the closure of $\alpha_{1}=W u_{m} \tau_{m-1}^{2 r_{m-1}+1} B_{t_{m}, m-1}=$ $W u_{m} \tau_{m-1} B_{t_{m}, m-1}$. By Lemma3 we obtain

$$
\alpha_{1}=W \tau_{m-1} v_{m-1} B_{t_{m}, m-1}
$$

where $v_{m-1}=A_{1, m-1}^{r_{1}} \ldots A_{m-2, m-1}^{r_{m-2}}$.

Figure 6 shows that there is only one crossing involving the $m$-strand in the braid $\alpha_{1}$. This is the occurrence of $\tau_{m-1}$. In $\hat{\alpha}_{1}$, we can get rid of the virtual crossing corresponding to $\tau_{m-1}$ with a virtual move (of type I). We have obtained a new link diagram $\hat{\alpha}_{2}$ where $\alpha_{2}=W v_{m-1} B_{t_{m}, m-1}$ has $m-1$ strands and $\hat{\alpha}_{2}$ is fused isotopic to $L$.

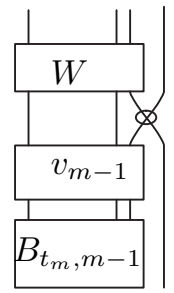

Figure 6: The braid $\alpha_{1}$

If we continue with this process, eventually we will get a braid $\beta$ in $B_{n}$ whose closure is fused isotopic to $L$. Note that each strand of $\beta$ corresponds to a different component of $L$ and therefore $\beta$ must be a pure braid. For $i<j$, define the group homomorphism $\delta_{i, j}: P B_{n} \rightarrow \mathbb{Z}$ by

$$
\delta_{i, j}\left(A_{s, t}\right)= \begin{cases}1 & \text { if } s=i \text { and } t=j \\ 0 & \text { otherwise. }\end{cases}
$$

Since $\beta$ is a pure braid it is easy to see that $\delta_{i, j}(\beta)=l k\left(\ell_{i}, \ell_{j}\right)$ where $\ell_{i}$ and $\ell_{j}$ are the corresponding components of $\hat{\beta}$.

This proves that any classical link $L$ with $n$-components can be obtained as the closure of a pure braid $\beta=x_{2} \ldots x_{n}$ where each $x_{k}$ can be written in the form $x_{k}=$ $A_{1, k}^{\delta_{1, k}} \ldots A_{k-1, k}^{\delta_{k-1, k}}$. This shows that $\beta$ depends only on the linking number of the components. 
Remark 1. We do not believe that the theorem will generalize to non-classical links where there are virtual crossings between different components. For example, let $U_{2}$ be the trivial link with two components and let $L=\hat{\alpha}$ where $\alpha=\sigma_{1} \tau_{1} \sigma_{1}^{-1} \tau_{1} \in F B_{2}$. Then both of these links have linking number 0 but we conjecture that they are not fused isotopic (although currently there are no known invariants to distinguish them).

[1] J. Birman, Braids, links and mapping class groups, Annals of Mathematical Studies 82, Princeton Univ. Press (1974).

[2] W.L. Chow, On the algebraic braid group. Annals of Math. 49 (1948), 654-658.

[3] R. Fenn, R. Rimányi and C. Rourke, The braid-permutation group, Topology 36 (1997), $123-135$.

[4] A. Fish and E. Keyman, Jones polynomial invariants, JKTR 15:3 (2006) 339-350.

[5] M. Goussarov, M. Polyak and O. Viro, Finite-type invariants of classical and virtual knots, Topology 39 (2000) 1045-1068.

[6] S. Kamada, Braid presentation of virtual knots and welded knots, arXiv:math.GT/0008092 v1.

[7] T. Kanenobu, Forbidden moves unknot a virtual knot, JKTR 10:1 (2001) 89-96.

[8] L.H. Kauffman, Virtual Knot Theory, European J. Combinatorics 20 (1999) 663-690. 